\title{
Synthesis of Antibodies-Conjugated Fluorescent Dye-Doped Silica Nanoparticles for a Rapid Single Step Detection of Campylobacter jejuni in Live Poultry
}

\author{
Wachira Tansub, ${ }^{1}$ Kooranee Tuitemwong, ${ }^{2}$ Pichet Limsuwan, ${ }^{3}$ \\ Supannee Theparoonrat, ${ }^{4}$ and Pravate Tuitemwong ${ }^{1}$ \\ ${ }^{1}$ Risk and Decision Analysis Laboratory (RADAL), Food Safety Center, KMUTT and Department of Microbiology, Faculty of Science, \\ King Mongkut's University of Technology Thonburi, Bangkok 10140, Thailand \\ ${ }^{2}$ Department of Microbiology, Faculty of Science, Kasetsart University, Bangkok 10900, Thailand \\ ${ }^{3}$ Department of Physics, Faculty of Science, King Mongkut's University of Technology, Thonburi, Bangkok 10140, Thailand \\ ${ }^{4}$ Department of Science Services, Ministry of Science and Technology, Bangkok 10400, Thailand \\ Correspondence should be addressed to Pravate Tuitemwong, ptt59@hotmail.com
}

Received 28 September 2011; Revised 28 December 2011; Accepted 29 January 2012

Academic Editor: Claude Estournes

Copyright ( 2012 Wachira Tansub et al. This is an open access article distributed under the Creative Commons Attribution License, which permits unrestricted use, distribution, and reproduction in any medium, provided the original work is properly cited.

The preparation of antibodies-conjugated fluorescent dye-doped silica nanoparticles (FDS-NPs) was developed to detect Campylobacter jejuni cells under a fluorescence microscope. The particles prepared by sol-gel microemulsion techniques have a round shape with an average size of $43 \pm 4 \mathrm{~nm}$. They were highly photo stable and could emit strong orange fluorescent for $60 \mathrm{~min}$. Both amine- and carboxyl-functionalized properties were evident from FTIR and FT Raman spectra. The FDS-NPs conjugated with antibodies against C. jejuni were well dispersed in PBS solution at $20 \mathrm{mM}$ of $\mathrm{NaCl}$. The conjugation with monoclonal antibodies against $C$. jejuni was successful. The direct observation of the antibodies-conjugated FDS-NPs- that bounds $C$. jejuni with Petroff Hausser counting chamber at 40x was clear. The different focus lengths clearly separated bound and unbound FDSNPs under the microscope. We successfully synthesis the bio-conjugated dye doped silica nanoparticles for C. jejuni that are easy to use and giving clear detection in due time.

\section{Introduction}

Campylobacteriosis is increasing and becomes one of the most frequently reported zoonotic diseases in humans worldwide. Its overall incidence was 54.6 cases per 100,000 inhabitants in the EU and 64.3 per 100,000 inhabitants in Belgium [1]. Its true incidence is uncertain since many unreported infections occur [2]. Ninety-one percent of human isolates in EU were identified as Campylobacter jejuni subsp. jejuni (hereafter $C$. jejuni), while Campylobacter coli accounts for most of the remaining cases. The heavy global burden of campylobacteriosis worldwide demands for development of more rapid, automatic, and sensitive detection methods. Traditional methods for the detection of pathogenic bacteria involve preenrichment, selective enrichment, biochemical screening, and serological confirmation [3]. This process is laborious and time consuming, especially for the bacteria like C. jejuni that requires special condition for growth. Many methods have been developed for rapid detection of pathogenic bacteria, including PCR [4], ELISA [5], piezoelectric biosensors [6], and amperometric biosensors [7].

Antibodies conjugated fluorescent dye-doped silica nanoparticles (FDS-NPs) have been developed for ultrasensitive bioassays [8-10]. Each nanoparticle encapsulates thousands of fluorescent dye molecules in a protective silica matrix, providing a highly amplified and reproducible signal for fluorescence-based bioanalysis. The bioconjugated nanoparticles enable significant amplification of the analytical signal compared to the conventional immunoassay. This is because of the many dye molecules inside each 


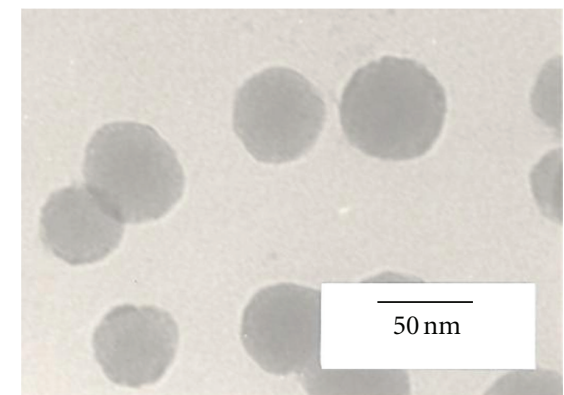

Figure 1: Transmission electron microscope images of FDS-NPs at 100,000x magnification.

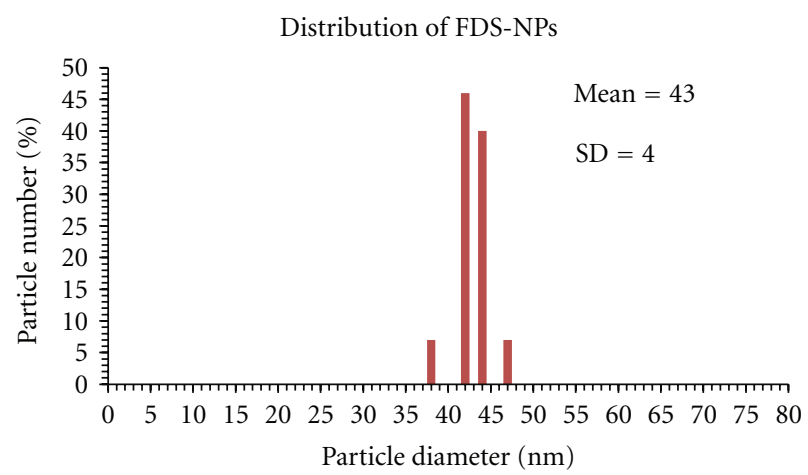

FIGURE 2: Size distribution of FDS-NPs produced by the sol-gel microemulsion method.

nanoparticle attached to the antibody molecule. There are many surface antigens available for specific recognition to the bacterium by using antibody-conjugated nanoparticles. Therefore, thousands of nanoparticles can bind to each bacterium, thereby producing a greatly amplified bright signal. The nanoparticles could be used in an assay tool, enabling the detection of one bacterium cell per given sample in a very short time (less than $20 \mathrm{~min}$ ) with a spectrofluorometer [9]. However, the high sensitivity provided by the fluorescence signal enhancement, selectivity, and reproducibility of nanoparticle-based bioassays can be diminished by the tendency of the silica nanoparticles to agglomerate irreversibly and cause nonspecific binding. These phenomena were due to the large surface area of the nanoparticles, as compared to those of dye molecules. The surface modification could be resulted in excess active functional groups, which are capable of binding to or interacting with various chemical and biological species and then lead to false positive signals. Thus, an important factor in the design of bioconjugated nanoparticles for the attachment to bacteria or biomolecules is to control covalent attachment of desired functional groups onto the particle surface [11]. To obtain successful and reproducible detection of biological targets using these fluorescent labels, the silica nanoparticles must be well dispersed in aqueous solution with minimal to no aggregation and nonspecific binding to biomolecules or substrates. The objective of this study was to establish synthesis and characterization of FDS-NPs without aggregation leading to the single-step detection of C. jejuni in bioconjugated dye-doped silica nanoparticles.

\section{Methodology}

2.1. Materials. The nanoparticles were prepared using microemulsion method modified from that reported by Bagwe et al. [11] and Suwanachart [12]. Briefly, tetraethylorthosilicate (TEOS), Triton X-100, Tris (2,2'-bipyridyl) dichlororuthenium(II) hexahydrate (Rubpy), succinic anhydride, $Z$-morpholinoethanesulfonic acid (Mes), BSA, 1-ethyl3-3(3-dimethylaminopropyl) carbodiimide hydrochloride, and $N$-hydroxysuccinimide were purchased from SigmaAldrich (USA). Trimethoxysilylpropyldiethylenetriamine, and $N$-(trimethoxysilylpropyl)-ethylenediamine were purchased from S.M. Chemicals (USA). Campylobacter jejuni was obtained from the Department of Medical Science, Ministry of Public Health, Thailand.

Ammonium hydroxide (28-30 wt\%), N,N-dimethylformamide, and affinity purified monoclonal mouse antiCampylobacter jejuni (mAbs) (isotype IgG1; clone 5E229) were purchased from Abcam (USA). It was supplied as a liquid in PBS (phosphate buffer saline), pH 7.2 with $0.1 \%$ sodium azide added. It was highly specific to $C$. jejuni and nonreactive with $H$. pylori, E. coli, Salmonella, Shigella, Yersinia, or Citrobacter. The antibody was stored at $-20^{\circ} \mathrm{C}$ until use.

2.2. Synthesis and Characterization of Silica Nanoparticles. The microemulsion solution was prepared by mixing $7.5 \mathrm{~mL}$ cyclohexane, $1.80 \mathrm{~mL}$-Hexanol, $1.77 \mathrm{~mL}$ Triton X-100, and $0.48 \mathrm{~mL} 20 \mathrm{mM}$ Rubpy dye in water for $20 \mathrm{~min}$ at room temperature. Then, $0.10 \mathrm{~mL}$ TEOS was added. Hydrolysis and condensation reactions were initiated by adding $0.09 \mathrm{~mL}$ $30 \% \mathrm{NH}_{4} \mathrm{OH}(30 \mathrm{wt} \%)$. The reaction was allowed to stir for $24 \mathrm{~h}$. After reaction was completed, one volume of acetone was added to break microemulsion and recover the particles. The particles were washed with 95\% ethanol (3 times) and deionized water (2 times). Ultrasonic bath was used to disperse the particles in the solutions. The particles were dried at $120^{\circ} \mathrm{C}$ for $2 \mathrm{~h}$ and stored in amber bottles.

The fluorescent dye-doped silica nanoparticles in 95\% ethanol were characterized by transmission electron microscope (TEM) (Jeol Model JEM-1230, USA) for the size, morphology, and distribution. The nanoparticles were also characterized for their excitation, emission, and photostability by spectrofluorometer technique (Nikon Model Eclipse E600, Japan). SEM EDS (Leo Model 1455P, Germany) was employed to study the particles compositions, and X-ray Diffractometer (Bruker Model D8 Advance, Germany, $40 \mathrm{kV}$, steptime 0.4 and stepsize 0.02 degree) was used for the analysis of the structure.

2.3. Determination of Aggregation of the Silica Nanoparticles. The $5 \mathrm{~mL}$ of particles $(10 \mathrm{mg} / \mathrm{mL})$ was mixed with $100 \mu \mathrm{g} / \mathrm{mL}$ antibody. The mixture was shaken regularly at $200 \mathrm{rpm}$ at $18^{\circ} \mathrm{C}$ for $2-4$ hours. Then, particles were washed in quenching solution once and centrifuged at $4500 \mathrm{rpm}$ at 


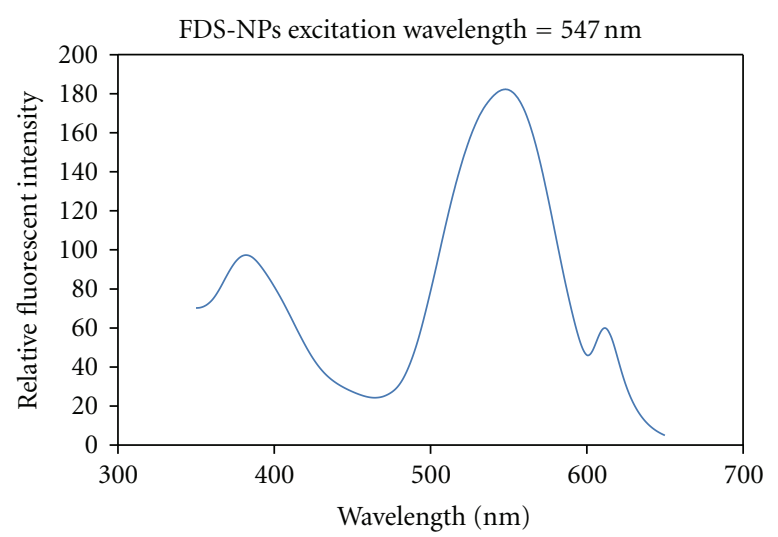

(a)

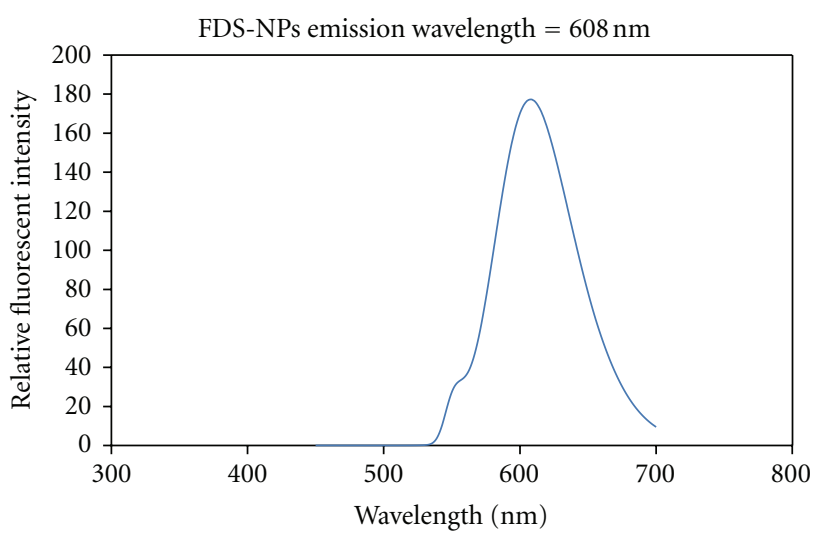

(b)

Figure 3: Excitation ((a); $547 \mathrm{~nm})$ and emission ((b); $608 \mathrm{~nm})$ wavelengths of the dye-doped silica nanoparticles.

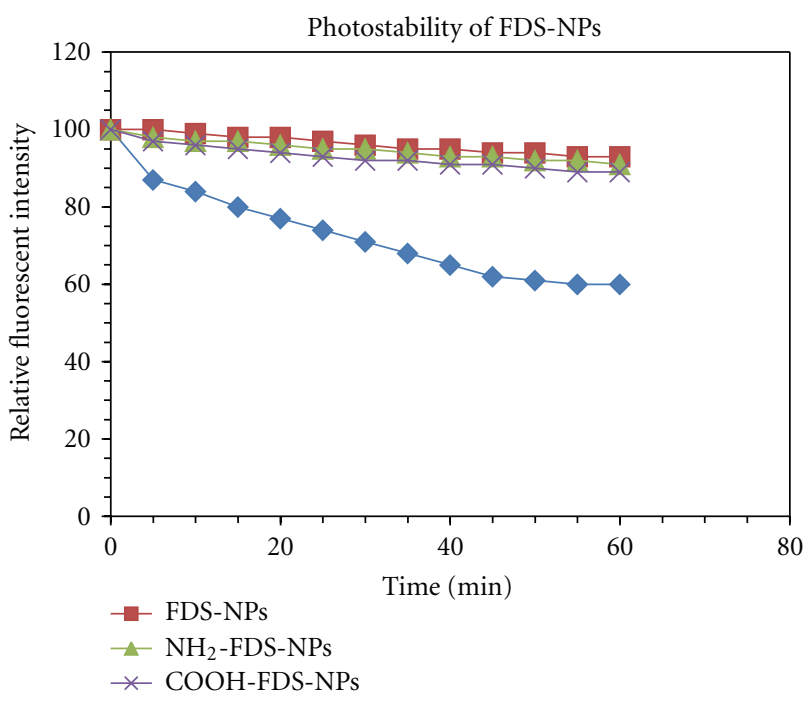

FIGURE 4: Fluorescent intensity of Rubpy dye, dye-doped nanoparticles, and surface-modified nanoparticles exposure to $150 \mathrm{~W}$ Xenon lamp excitation.

$18^{\circ} \mathrm{C}$ for $10 \mathrm{~min}$. They were distributed in $5 \mathrm{~mL}$ quenching solution and regularly shaken at $18^{\circ} \mathrm{C}$ for $30 \mathrm{~min}$. Then they were washed with the storage buffer 1 time and centrifuged at $4500 \mathrm{rpm}$ at $18^{\circ} \mathrm{C}$ for $10 \mathrm{~min}$. The particles were dispersed in $\mathrm{NaCl}$ solution at concentrations of 5, 10, 15, 20, $25 \mathrm{mM}$. The particles were then analyzed for zeta potential by the NanoSizer machine at the Department of Physics, Kasetsart University, Thailand.

2.4. Chemical Modification of the Nanoparticle Surface. The surface of FDS-NPs that was chemically modified before mAbs against $C$. jejuni was immobilized onto the nanoparticles. The methods were modified from that reported by Zhao et al. [9], Bagwe et al. [11], and Suwanachart [12].

Amine-functionalized group was added on the nanoparticle surfaces using $32 \mathrm{mg}$ of silica nanoparticles washed with deionized water (2 times) and $1 \mathrm{mM}$ acetic acid (once).
The particles were dispersed with ultrasonication and reacted with $20 \mathrm{~mL}$ of $10 \%$ (3-aminopropyl) trimethoxysilyl propyldiethylenetriamine in $1 \mathrm{mM}$ acetic acid (APTS) for $30 \mathrm{~min}$ at room temperature $\left(25^{\circ} \mathrm{C}\right)$ with continuous stirring. The particles were stored in $0.1 \mathrm{M} \mathrm{PBS}\left(\mathrm{pH} \mathrm{7.3)}\right.$ at $4^{\circ} \mathrm{C}$.

The amine-functionalized nanoparticles were then thoroughly washed three times by deionized water and washed with $N, N$-dimethylformamide and further reacted with $10 \%$ succinic anhydride in $N, N$-dimethylformamide solution under nitrogen gas for $6 \mathrm{~h}$ with continuous stirring. The particles were analyzed to verify the attachment of the functional groups on the surface using Fourier Transformed mid Infrared Spectrophotometer (range of $4500-450 \mathrm{~cm}^{-1}$ resolution $4.0 \mathrm{~cm}^{-1}$, No of Scan 16) (FTIR, Perkin Elmer Model Spectrum One, USA) and FT-Raman (750 mW, Res $4.0 \mathrm{~cm}^{-1}$, no of scan 1000; Bruker IFS $66 \mathrm{v} / \mathrm{s}$, USA).

2.5. Immobilization of Antibodies on Nanoparticle Surface. An aliquot of $5 \mathrm{~mL}$ of $0.1 \mathrm{mg} / \mathrm{mL}$ nanoparticles was reacted with $2 \mathrm{~mL}$ of $5 \mu \mathrm{g}$ protein/mL of the antibody for $2-4 \mathrm{~h}$ at room temperature with continuous stirring, followed by washing with $0.1 \mathrm{M}$ PBS buffer $\mathrm{pH}$ 7.3. The antibody-conjugated nanoparticles surface was blocked with $1 \%$ BSA and washed in $0.1 \mathrm{M}$ PBS before use.

\section{Results and Discussion}

3.1. Preparation and Characterization of Silica Nanoparticles. The W/O microemulsion and sol-gel techniques were used for the preparation of nanoparticles. Sizes were collected from 30 particles from TEM image (Jeol Model JEM1230, USA) to evaluate mean and standard deviation of the particles using Program Origin 7 (USA, 1991-2002). It was found that the distribution of diameter of particles was in the range of $38 \mathrm{~nm}$ to $47 \mathrm{~nm}$ with the average and standard deviation of $43 \pm 4 \mathrm{~nm}$. The particles were round in shape with narrow size distribution and well dispersed as shown in Figure 1.

The particles of with diameter of $42 \mathrm{~nm}$ and $44 \mathrm{~nm}$ were $40 \%$ and $46 \%$, respectively. They were accounted for $86 \%$ 


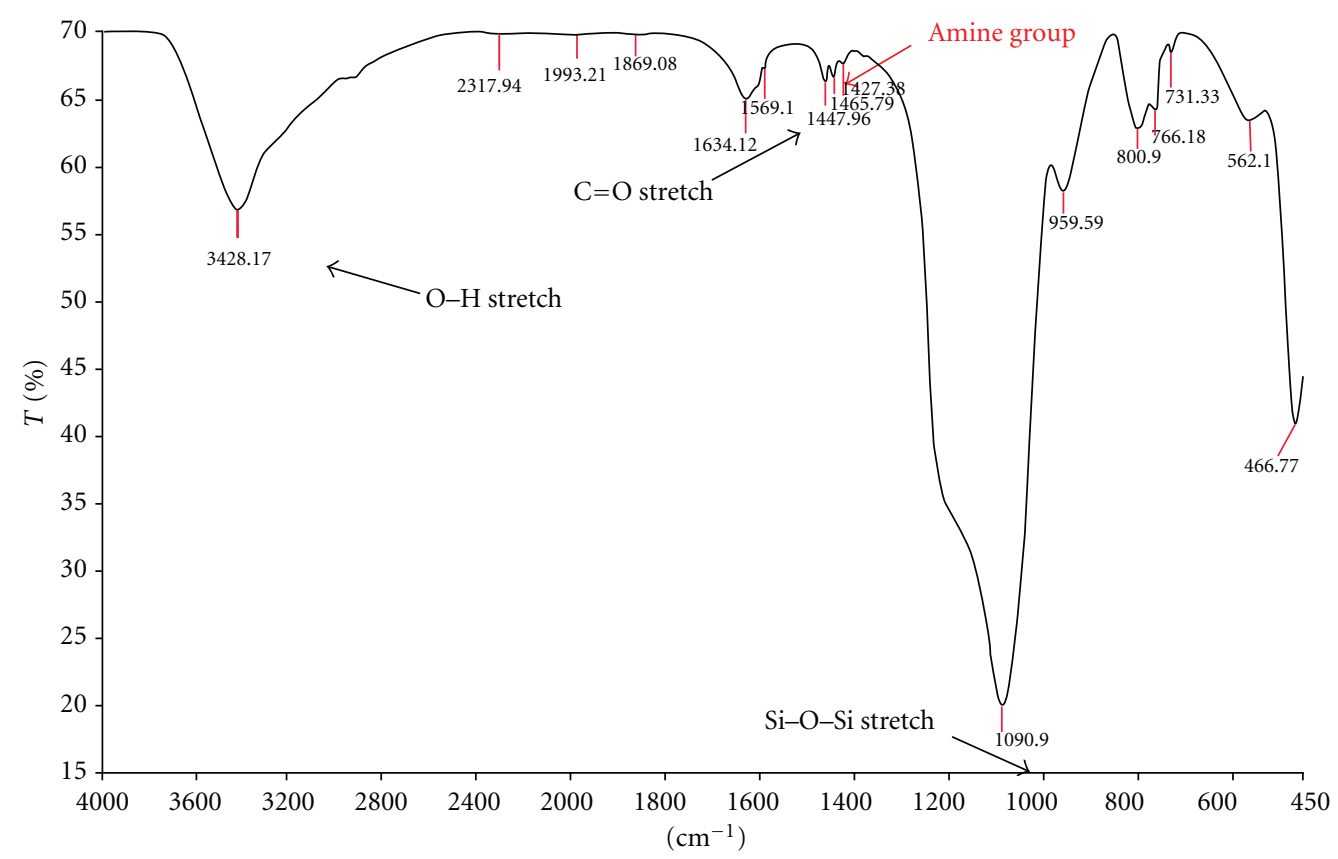

FIGURE 5: FTIR spectrum of FDS-NPs with amine modification.

of total particles. Particles of size 38 and $47 \mathrm{~nm}$ diameter contained only 14\% (Figure 2).

The aqueous dye molecules were trapped inside a silica matrix to form the fluorescent dye-doped silica nanoparticles. Spectrofluorometric measurements of the excitation and the emission spectra of the nanoparticles were carried out in the aqueous solution. They exhibited maximum emission at $608 \mathrm{~nm}$ when excited at the $547 \mathrm{~nm}$ (Figure 3). The excitation spectra were the same for the Rubpy dye and nanoparticles, but the maximum emission band of the Rubpy dye shifted by 7 and $8 \mathrm{~nm}$, respectively, to the longer wavelength compared with the nanoparticles luminescence.

Photobleaching of Rubpy dye, fluorescent dye-doped silica nanoparticles and surface-modified nanoparticles, were studied under a long period of continuous intensive exposure $(60 \mathrm{~min})$ to a 150 watt xenon lamp light. The results showed that free Rubpy dye had photobleaching of about $40 \%$ after $60 \mathrm{~min}$, while those of the particles (particles with and without additional functional groups) were much more stable (Figure 4 ). It was clearly indicated that silica coating of the nanoparticles was able to minimize photobleaching of the luminescence dyes. Silica coating appeared to completely prevent outside oxygen molecule from penetration and reaction with inside dye molecules which cause photobleaching [13].

3.2. Surface Modification of FDS-NPs. Figures 5-7 displayed FTIR and FT-Raman spectra of FDS-NPs. It was found that $\mathrm{C}=\mathrm{O}$ stretch (Carboxyl group) and $\mathrm{N}-\mathrm{H}$ (amine stretch) did not clearly shown in FTIR spectra (Figure 5). The existence of amino and carboxyl groups was evident in FT-Raman spectra in Figures 6 and 7, respectively. The FT-Raman spectrum showed hydroxyl group $(\mathrm{O}-\mathrm{H})$ at $1,150-1,170$
Raman shift $\mathrm{cm}^{-1}$, silica ( $\mathrm{Si}-\mathrm{O}-\mathrm{Si}$ ) at 650 Raman shift $\mathrm{cm}^{-1}$, carboxyl $(\mathrm{C}=\mathrm{O})$ at 1,550-1,660 Raman shift $\mathrm{cm}^{-1}$. The other carboxyl group in carboxyl functionalized nanoparticles was also indicated at $1,653.14 \mathrm{~cm}^{-1}$. The findings confirmed the success modifications of FDS-NPs surfaces. The functional group that was for the antibodies to attach onto is surface.

The carboxyl group could be seen at the peak at $1,653.14 \mathrm{~cm}^{-1}$ under FTIR spectrum. The attachment of $C$. jejuni monoclonal antibodies on the modified surface FDSNPs was a success and the particles could react with the target C. jejuni as designed (Figure 8).

3.3. Reduction of the Aggregation of Silica Nanoparticles. However, after amine modification, the surface of FDSNPS became positively charged [14]. The phenomena caused agglutination of the particles. The positive charge on surface of the particle was not consistent and attracted the negativecharged particles resulted in aggregation of the particles after surface modification. TEM images of amine- and carboxylmodified nanoparticles indicated that some particles were aggregated.

After amine modification, FDS-NPS were dispersed with sonication probe. The particles then went through carboxyl modification with succinic anhydride. The process modified the surfaces of particles to negative charge and hence reduced the agglomeration of the particles. Succinic anhydride produced negative charges and caused repulsion among the particles.

Effects of sodium chloride at various concentrations on aggregation of antibody-conjugated FDS-NPS were shown in Figure $8 . \mathrm{NaCl}$ at 5, 10, 15, 20, $25 \mathrm{mM}$ in PBS buffer caused the zeta potentials to decrease from -6 to $-40 \mathrm{mV}$. Results from Nanosizer analysis, a light scattering detector for the 


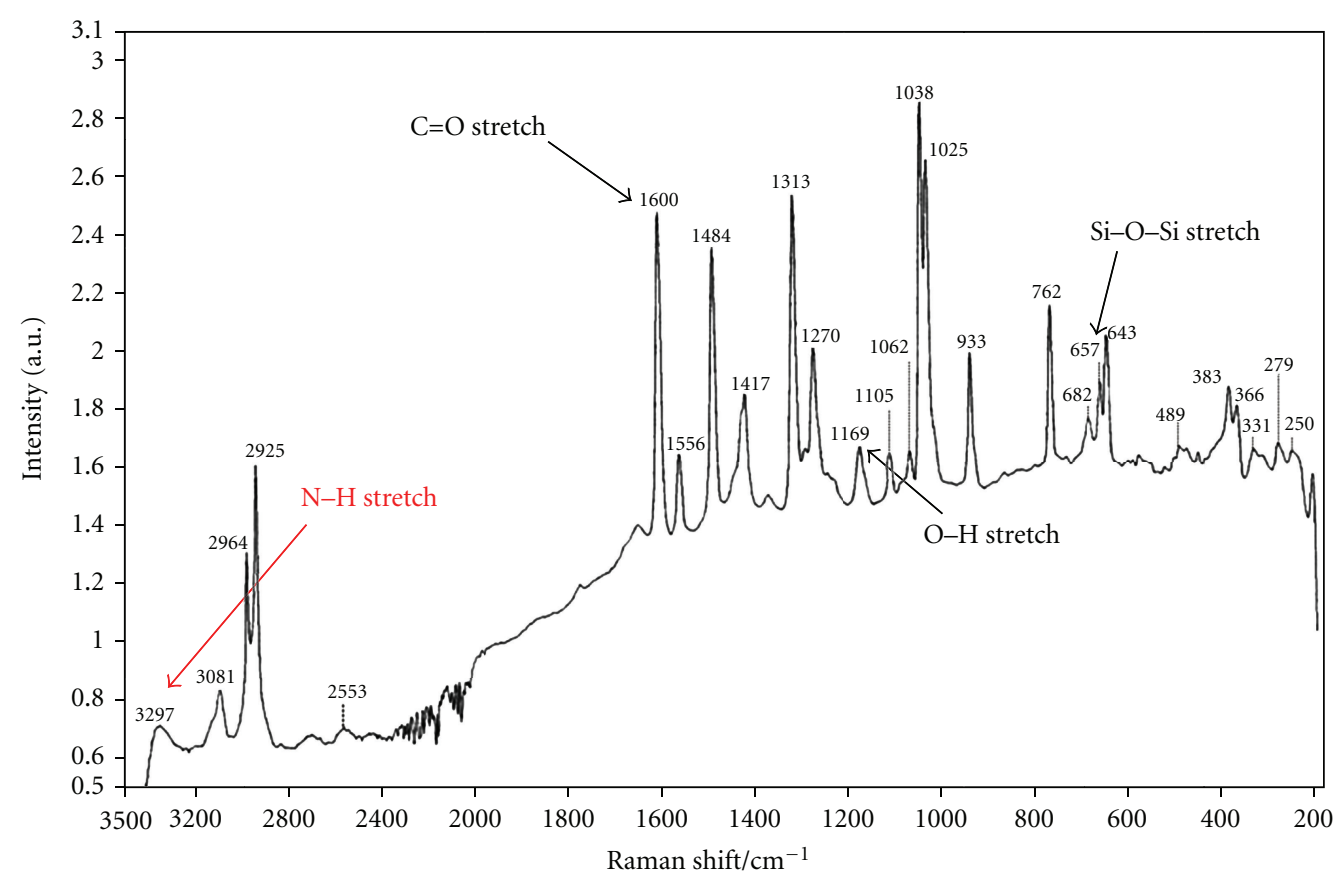

FIgURE 6: FT-Raman spectrum of FDS-NPs with amine modifications.

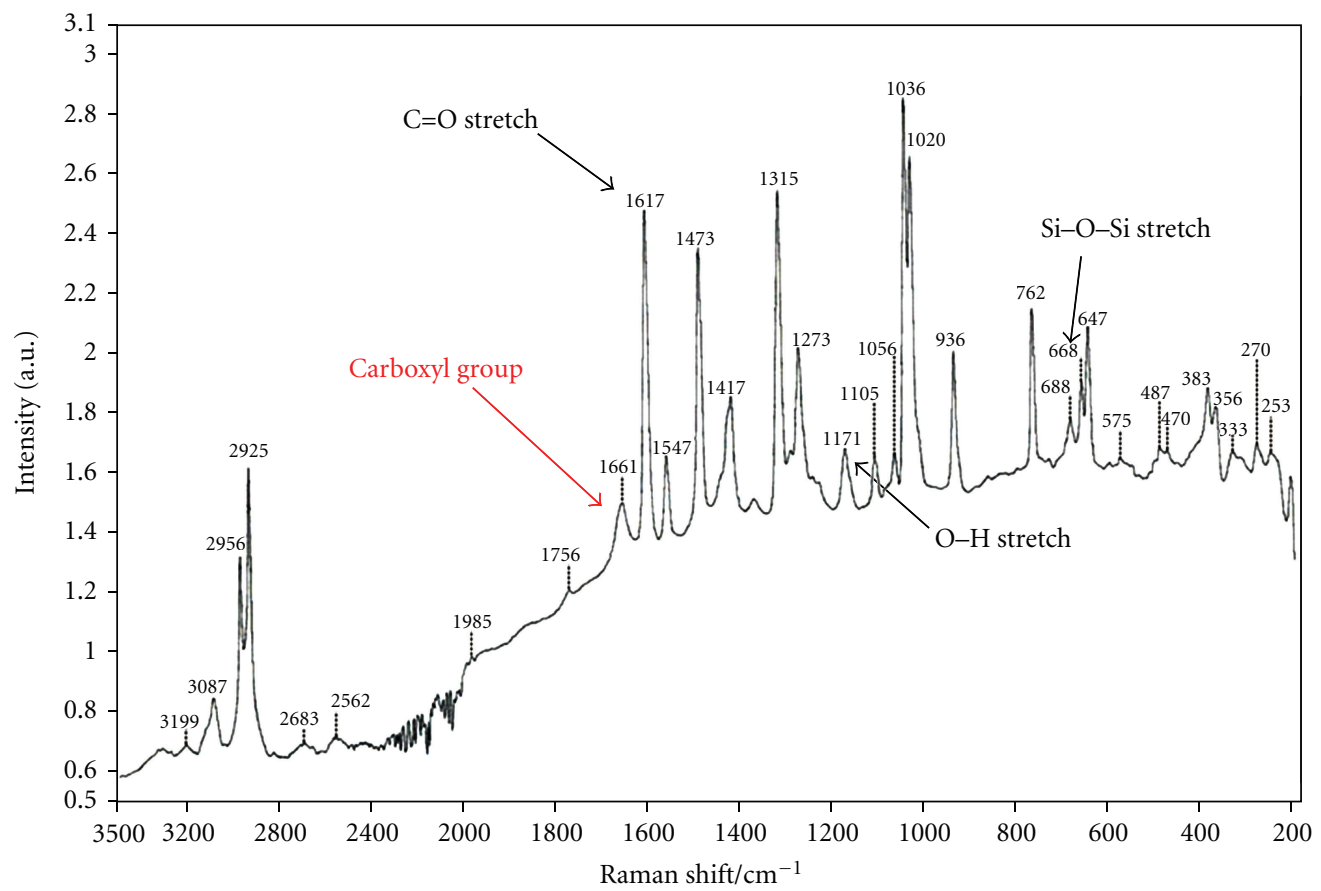

Figure 7: FT-Raman spectrum of FDS-NPs with carboxyl modification.

prediction of dispersion stability, showed the zeta-potential of between -30 to $30 \mathrm{mV}$ indicating that the suspension was not stable. The values outside -30 to $30 \mathrm{mV}$ indicated stable condition which means the particles dispersed evenly and did not form aggregates.

In this study, the concentrations of $\mathrm{NaCl}$ from $10 \mathrm{mM}$ to $25 \mathrm{mM}$ gave zeta-potential value of less than $-30 \mathrm{mV}$.
The $\mathrm{NaCl}$ concentration at $10 \mathrm{mM}$ that gave the values lower than -30 resulted in less aggregation allowing antibodies coated on the surface to function properly. Higher concentration could affect the activities of the antibodies [15].

Figure 9 demonstrated that $C$. jejuni surrounded with antibody-conjugated FDS-NPs giving the bright color appearance under epifluorescence microscope (40x). 


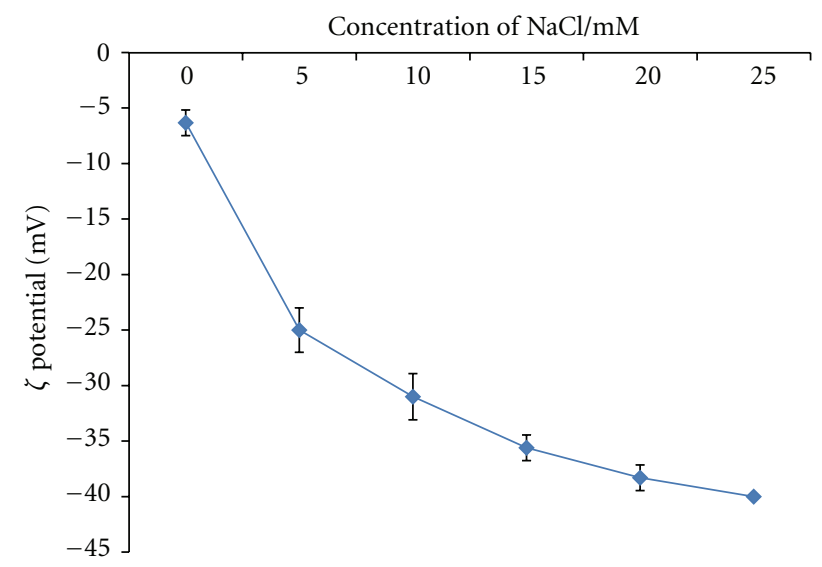

FIgURE 8: Effects of $\mathrm{NaCl}$ concentrations on zeta potential $(\mathrm{mV})$ of the silica nanoparticles.

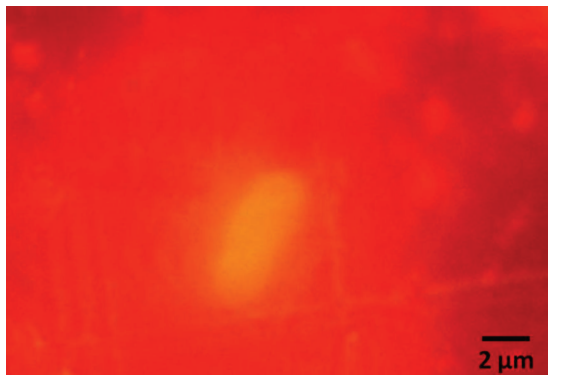

Figure 9: Antibody-conjugated FDS-NPs attached to C. jejuni cell surface in Petroff-Hausser counting chamber under epifluorescent microscope (40x).

The sample was placed in a Petroff-Hausser counting chamber $(0.05 \times 0.05 \times 0.2 \mathrm{~mm})$.

The depth of chamber appeared to have different focus lengths of small particles and larger cells coated with a large number of FDS-NPs. Cells surrounded with the FDS-NPs were detected with a shorter focusing length while those unattached were smaller and sank to bottom hence required longer distance to be focused. With this technique, the $C$. jejuni cells were distinctively bright orange color while the background was clearly seen as sediments of unattached dispersed particles and small aggregates compared with coated cells.

\section{Conclusion}

A water-in-oil microemulsion in cooperation with solgel techniques and surface modification method was used to prepare uniform size of fluorescent dye-doped silica nanoparticles. The application of sodium chloride could reduce aggregates of the antibody conjugated silica nanoparticles. In this contribution, a homogenous noncompetitive immunoassay has been developed based on the aggregation of antibody-functionalized silica nanoparticles that could be observed under epifluorescent microscope. Using Petroff-Hausser counting chamber successfully separated cells attached with nanoparticles from the unattached particles.

\section{Acknowledgments}

This research was funded by the Ministry of Science and Technology, Royal Government of Thailand and the National Research Council of Thailand (NRCT).

\section{References}

[1] EFSA, "The Community Summary Report on Trends and Sources of Zoonoses, Zoonotic Agents, Antimicrobial Resistance and Foodborne Outbreaks in the European Union in 2006," The EFSA Journal, 130 pages, 2007.

[2] WHO, "The Increasing Incidence of Human Campylobacteriosis. Report and Proceedings of a WHO Consultation of Experts," WHO/CDS/CSR/APH/2000.4., 2000.

[3] D. Ivnitski, I. Abdel-Hamid, P. Atanasov, and E. Wilkins, "Biosensors for detection of pathogenic bacteria," Biosensors and Bioelectronics, vol. 14, no. 7, pp. 599-624, 1999.

[4] D. Rodríguez-Lázaro, M. D’Agostino, A. Herrewegh, M. Pla, N. Cook, and J. Ikonomopoulos, "Real-time PCRbased methods for detection of Mycobacterium avium subsp. paratuberculosis in water and milk," International Journal of Food Microbiology, vol. 101, no. 1, pp. 93-104, 2005.

[5] B. W. Brooks, J. Devenish, C. L. Lutze-Wallace, D. Milnes, R. H. Robertson, and G. Berlie-Surujballi, "Evaluation of a monoclonal antibody-based enzyme-linked immunosorbent assay for detection of Campylobacter fetus in bovine preputial washing and vaginal mucus samples," Veterinary Microbiology, vol. 103, no. 1-2, pp. 77-84, 2004.

[6] Y. Y. Wong, S. P. Ng, M. H. Ng, S. H. Si, S. Z. Yao, and Y. S. Fung, "Immunosensor for the differentiation and detection of Salmonella species based on a quartz crystal microbalance," Biosensors and Bioelectronics, vol. 17, no. 8, pp. 676-684, 2002.

[7] S. Susmel, G. G. Guilbault, and C. K. O'Sullivan, "Demonstration of labeless detection of food pathogens using electrochemical redox probe and screen printed gold electrodes," Biosensors and Bioelectronics, vol. 18, no. 7, pp. 881-889, 2003.

[8] S. Santra, P. Zhang, K. Wang, R. Tapec, and W. Tan, "Conjugation of biomolecules with luminophore-doped silica 
nanoparticles for photostable biomarkers," Analytical Chemistry, vol. 73, no. 20, pp. 4988-4993, 2001.

[9] X. Zhao, R. Tapec-Dytioco, K. Wang, and W. Tan, "Conjugation of biomolecules with luminophore-doped silica nanoparticles for photostable biomarkers," Analytical Chemistry, vol. 75, pp. 3476-3483, 2003.

[10] X. Zhao, R. Tapec-Dytioco, and W. Tan, "Ultrasensitive DNA detection using highly fluorescent bioconjugated nanoparticles," Journal of the American Chemical Society, vol. 125, no. 38, pp. 11474-11475, 2003.

[11] R. P. Bagwe, C. Yang, L. R. Hilliard, and W. Tan, “Optimization of dye-doped silica nanoparticles prepared using a reverse microemulsion method," Langmuir, vol. 20, no. 19, pp. 83368342, 2004.

[12] T. Suwanachart, Preparation of bioconjugated fluorescent-doped silica nanoparticles for the detection of Vibrio cholera O139, M.S. thesis, Applied Microbiology Program, Department of Microbiology, Faculty of Science King Mongkut's University of Technology Thonburi, Thailand, 2008.

[13] X. Hun and Z. Zhang, "Functionalized fluorescent core-shell nanoparticles used as a fluorescent labels in fluoroimmunoassay for IL-6," Science Direct, vol. 22, pp. 1743-1748, 2007.

[14] R. P. Bagwe, L. R. Hilliard, and W. Tan, "Surface modification of silica nanoparticles to reduce aggregation and nonspecific binding," Langmuir, vol. 22, no. 9, pp. 4357-4362, 2006.

[15] B. Du, Z. Li, and Y. Cheng, "Homogeneous immunoassay based on aggregation of antibody-functionalized gold nanoparticles coupled with light scattering detection," Talanta, vol. 75, no. 4, pp. 959-964, 2008. 

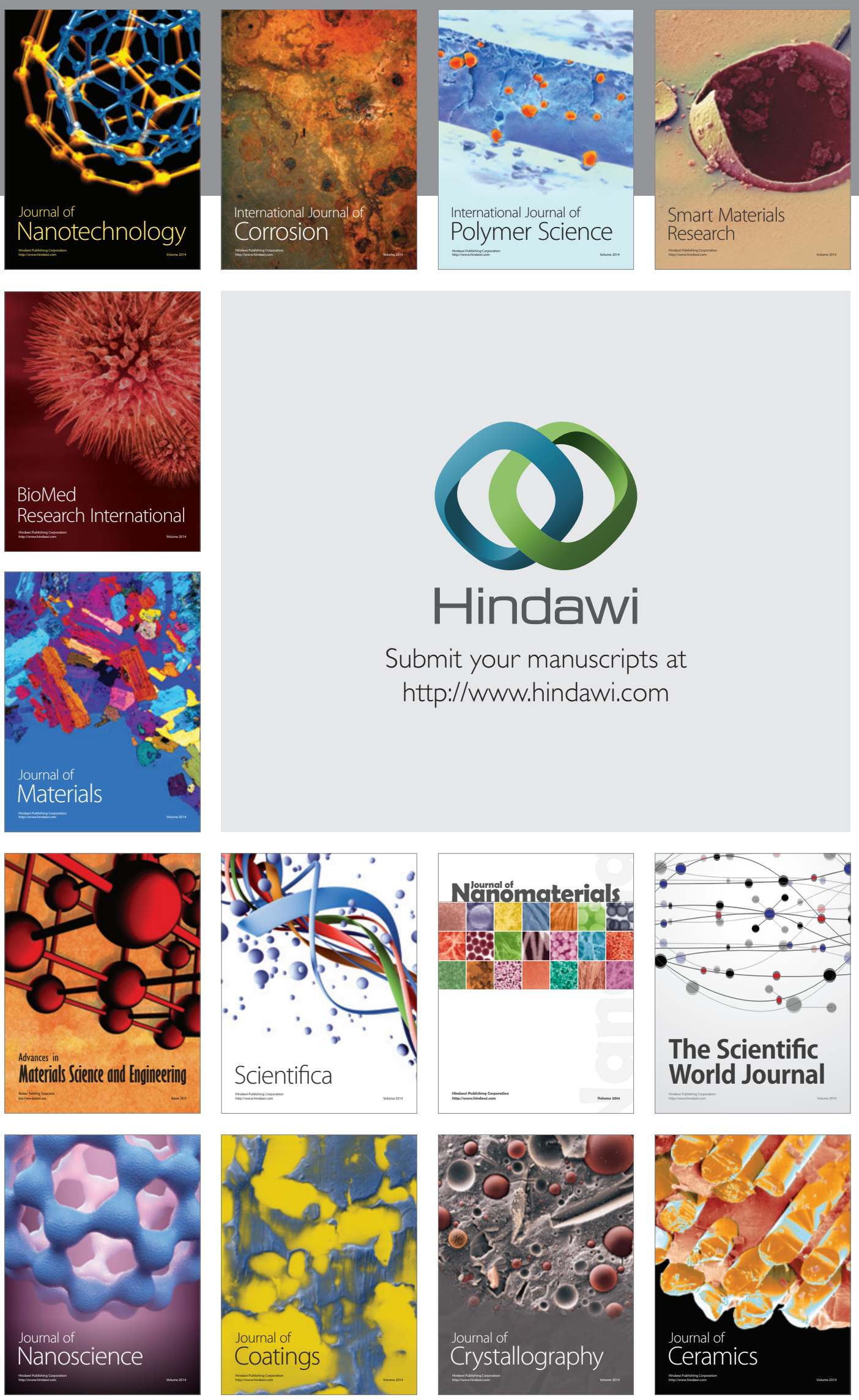

The Scientific World Journal

Submit your manuscripts at

http://www.hindawi.com

\section{World Journal}

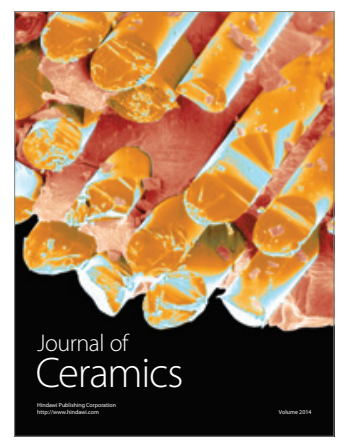

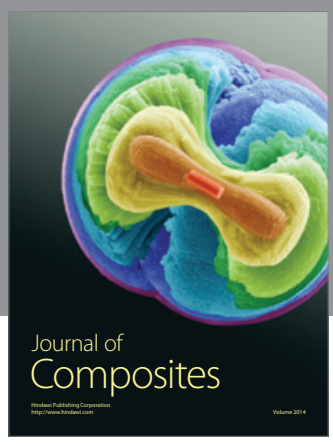
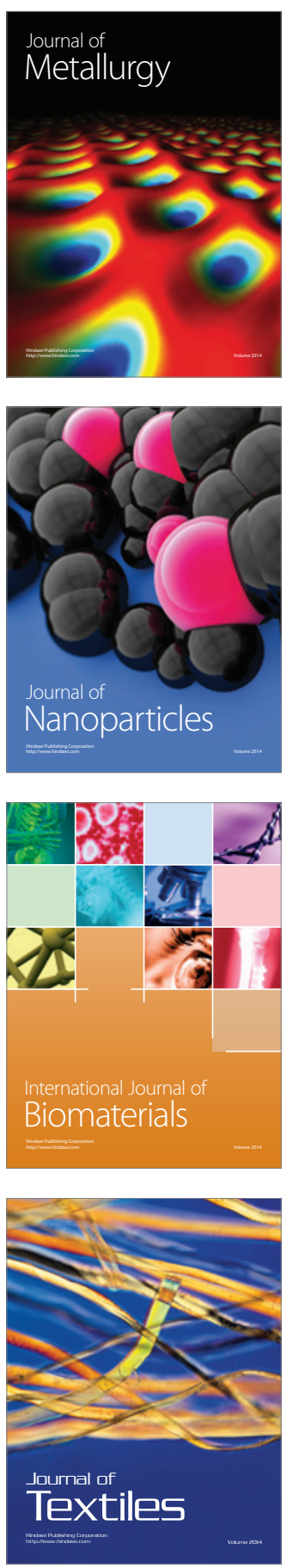